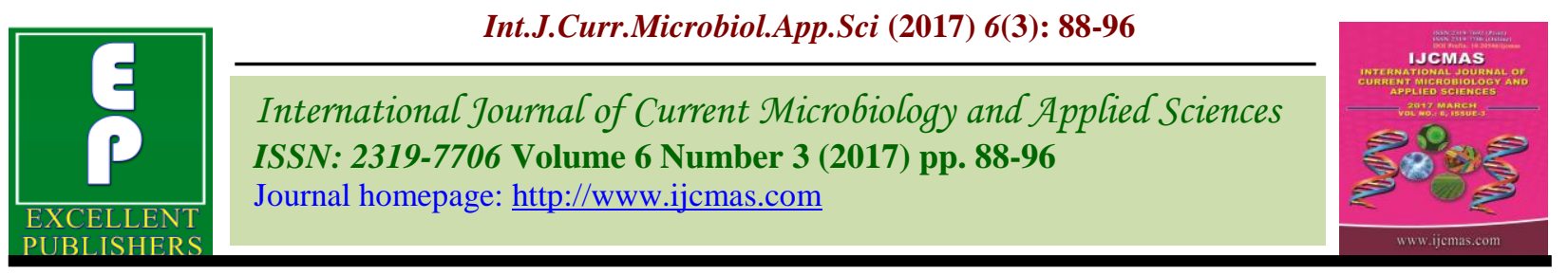

Original Research Article https://doi.org/10.20546/ijcmas.2017.603.009

\title{
Fertility Status of Irrigated Soils of Jhotwara Panchayat Samiti of Jaipur District, India
}

\author{
Kamla Kumari Choudhary ${ }^{1}$, B.L. Yadav $^{2}$, K.K. Sharma ${ }^{2}$, R.D. Jat ${ }^{3}$ and S.K. Kakraliya ${ }^{4}$ \\ ${ }^{1}$ Department of Soil Science, Central Arid Zone Research Institute, RS Pali Rajasthan, India \\ ${ }^{2}$ SKN, Agricultural University- Jobner, Rajasthan, India \\ ${ }^{3}$ International Maize and Wheat Improvement Center, India \\ ${ }^{4}$ CCS Haryana Agriculture University, Hissar125004, India \\ *Corresponding author
}

\begin{tabular}{|c|c|}
\hline & A B S T R A C T \\
\hline $\begin{array}{l}\text { Ke y w o r d s } \\
\text { Fertility status, } \\
\text { Available } \\
\text { micronutrients, } \\
\text { Jhotwara Panchayat } \\
\text { Samiti of Jaipur. }\end{array}$ & \multirow{3}{*}{$\begin{array}{l}\text { The present investigation entitled "Fertility status of irrigated soils of Jhotwara } \\
\text { panchayat samiti of Jaipur district "was carried out during } 2010-11 \text {. The soils } \\
\text { were } \mathrm{pH} \text { neutral to alkaline }(\mathrm{pH} 7.2-8.5) \text { in reaction having electrical conductivity } \\
\text { (EC) value in surface soil }\left(0.53-2.51 \mathrm{dSm}^{-1}\right) \text {. The data on nutrient showed that the } \\
\text { soils are low in organic carbon and available nitrogen and low to medium in } \\
\text { available phosphorus and available sulphur whereas, medium to high in available } \\
\text { potassium. Among the DTPA extractable micronutrient cations, manganese }(\mathrm{Mn}) \\
\text { and copper }(\mathrm{Cu}) \text { were found to be above critical limits, whereas the soils were } \\
\text { deficient in iron }(\mathrm{Fe}) \text { and zinc ( } \mathrm{Zn}) \text { supply. }\end{array}$} \\
\hline Article Info & \\
\hline $\begin{array}{l}\text { Accepted: } \\
\text { 08 February } 2017 \\
\text { Available Online: } \\
10 \text { March } 2017\end{array}$ & \\
\hline
\end{tabular}

\section{Introduction}

A systematic study of soils and water is necessary for better utilization of land and water resources and to tackle soil and water problems. All detailed information is not yet available about soil fertility status and quality of irrigation water of this tract which is essential for an effective land management. Therefore, an urgent need was felt for extensive and well planned investigation both in the field and laboratory for suggesting guidelines towards better utilization of soil and irrigation water of this tract. Recommendation of fertilizer based on soil and water analysis will be easy and more appropriate for economic productivity. Availability of N, P, K, secondary and micro nutrients may induce better germination of seeds and hence subsequent better growth and stronger root development may make the plants more capable of tolerating salt stress particularly at later stages of solution and adjust their leaf water potential and thereby internal resistance against salinity/sodicity. The diagnosis of salinity, sodicity and fertility status of the soil and water resources along with their nutritional enrichment is of vital significance. Potassium and calcium have been reported to counteract the adverse effect on sodium in soil. The most important constituents in soil is organic matter, an appreciable amount of it in soil tremendously increase soil fertility. Decay of organic matter 
release nitrogen, phosphorus and mineral nutrients in forms available to plant. Organic carbon is also positively correlated with total and available nitrogen in all soil group (Verma et al., 1980). Micronutrients are also essential for crop growth but are not regularly applied in the soil along with the common fertilizer used by the farmers. Their removal from the soil had been for centuries without any systematic replenishment. Micronutrient deficiencies were first reported at the end of the 19th century and today it is well know that the extensive areas of our soils are incapable of supplying plants with sufficient amount of micronutrients. The application of high analysis NPK fertilizers in the soil having only major nutrients, the loss of micronutrients though plant uptake and leaching, the decreasing proportion of farm yard manure (FYM) and other organic manures in comparison with fertilizer and several other factors collectively contribute towards the deficiency of micronutrients in soils. Therefore, the data will help in delineating the area of deficiency and sufficiency.

\section{Materials and Methods}

The study area was undertaken in Jhotwara Panchayat samiti is located in Jaipur district and lies under zone III-A (semi arid eastern plain) of Rajasthan. It is situated between $26^{\circ} 23^{\prime}$ and $27^{\circ} 51^{\prime}$ North latitude and $74^{\circ} 55^{\prime}$, and 75050 East longitude with an area of $11152 \mathrm{~km}^{2}$. The climate of the area is semiarid. In summer maximum temperature varies from 38 to $45^{\circ} \mathrm{C}$ whereas in winter generally minimum temperature lies between 5 and 18 ${ }^{0} \mathrm{C}$. The mean annual rainfall of the locality is $450 \mathrm{~mm}$, most of this is received during monsoon season. Main source of irrigation is tube well. A maximum 52 to $58^{\circ} \mathrm{C}$ soil temperature has been recorded at surface during summer months. Eighty six composite surface (0-0.15 $\mathrm{m}$ depth) soil samples of cultivated fields irrigated with tube well water were collected from 86 villages of Jhotwara panchayat samiti. Approximately $2 \mathrm{~kg}$ of soil was taken by khurpi in each cloth bags. Samples were air dried ground and passed through $2 \mathrm{~mm}$ sieve and stored in properly labeled plastic bottles. Soil samples were collected before Rabi crop season in the year 2010. The soil reaction $(\mathrm{pH})$ and electrical conductivity (EC) were determined in saturation extract as per procedure described by Jackson (1973). The soil organic carbon (OC) was estimated by wet digestion method of Walkley and Black (1934) and the available $\mathrm{N}$ of the soils was determining by Subbaiah and Asija (1956). The available P in the soil was extracted by employing Olsen extractant (0.5 $M$ NaHCO3) as described by Olsen et al., (1954) and available $\mathrm{K}$ in the soils was extracted by using neutral ammonium acetate and the content was determine by aspirating the extract into flame photometer (Jackson, 1973). The available sulphur $(\mathrm{S})$ in the soils was extracted with $0.15 \% \mathrm{CaCl} 2.2 \mathrm{H}_{2} \mathrm{O}$ solution as described by Williams and Steninbergs (1959) and the content of DTPA extractable micronutrient viz., iron $(\mathrm{Fe})$, manganese $(\mathrm{Mn})$, copper $(\mathrm{Cu})$ and zinc $(\mathrm{Zn})$ in soil was estimated using 1:2 soil suspension to exttractants ratio (Lindsay and Norvell, 1978).

\section{Results and Discussion}

\section{Soil chemical properties}

The chemical properties of the soil revealed that it is neutral to alkaline, the $\mathrm{pH}$ range from 7.2 to 8.5 (surface) (Table 1). The highest value of $\mathrm{pH} 8.5$ ) was recorded at Panchayat in Durjiniwas, Begus, Dhankiya, Chakbasdi and lowest value of (pH7.2) was observed at Dhankiya Panchayat. $\mathrm{pH}$ of study area were varied from normal to alkaline. Accumulation of bases especially $\mathrm{Na}^{+}$under low rainfall conditions seen to be the primary reason for alkaline soil reaction. The variation of $\mathrm{ph}$ 
from neutral to alkaline has also been reported by Mathur et al., (2006), Meena et al., (2006) in the soils of Rajasthan state. With the increasing of soil ph the availability of micronutrient decreased, which probably due to alkaline nature of soils. This confirmed that the soil $\mathrm{pH}$ had a significant and negative correlation with available $\mathrm{Fe}\left(\mathrm{r}=-0.423^{* *}\right)$, $\mathrm{Mn}(\mathrm{r}=-0.477 * *), \mathrm{Cu}\left(\mathrm{r}=-0.461^{* *}\right)$ and $\mathrm{Zn}$ $\left(\mathrm{r}=-0.448^{* *}\right)$ (Table 2). The neutral to alkaline $\mathrm{pH}$ may be attributed to the reaction of applied fertilizer material with soil colloids, which resulted in the retention of basic cations on the exchangeable complex of the soil. Similar trend was reported by Sharma et al., (2008). The electrical conductivity of surface soils was ranged between 0.53 to 2.51 with the mean value of 1.27. A perusal of data mentioned in table 1 . The lowest values $(0.53 \mathrm{dS} / \mathrm{m})$ were recorded in the soil of Chakbasdi Panchayat, whereas, the highest value $(2.51 \mathrm{dS} / \mathrm{m})$ was observed in soil of Sumel Panchayat. Majority of the soils (93.02\%) have ECe less than $2 \mathrm{dS} / \mathrm{m}$ and could be regarded as non-saline soils. Such results were also reported by Sumner (1995), Sharma et al., (2004)

\section{Nutrient status}

All the soil samples collected (surface soil) from different sites of the studied area were analysed for organic carbon, available nitrogen, available phosphorus, available potassium, available sulphur, available zinc, available iron, available copper and available manganese are presented in table 1. The organic carbon content not only plays an important role in increasing cation exchange capacity, aggregation, water holding capacity and fertility status of soils but also contributes to a great deal, both directly and indirectly in influencing many physico-chemical properties of soils. Organic carbon varied from 0.10 to 0.40 per cent with the minimum value $(0.10$ per cent) at Begus Panchayat and the maximum value ( 0.40 per cent) at Nemera Panchayat. All the soil samples fall under low category of organic carbon. The low organic carbon content of these soils might be due to the absence of stable aggregate (Jolivet et al., 1997), sever wind erosion (Wu and Tiessen, 2002), high microbial decay scanty natural vegetations and poor decomposition due to low rainfall and rapid oxidation due to high summer temperature. Available nitrogen of these soils varied between 122.07 to 198.71 $\mathrm{kg} / \mathrm{ha}$ with a lowest value at Begus Panchayat and highest at Nemera Panchayat. Soils having available nitrogen less than $250 \mathrm{~kg} / \mathrm{ha}$ could be classified as low in available nitrogen (Subbaih and Asija, 1956). From the data it is clear that all the soils were low in available nitrogen. Low available nitrogen might be due to presence of high sand particles and low organic matter content. Soil available $\mathrm{N}$ had significant and positive correlation with OC $(\mathrm{r}=0.989 * *)$. The available phosphorus content of surface soil samples varied between 15 to $47 \mathrm{~kg} / \mathrm{ha}$. The maximum value $\left(47 \mathrm{Kg} \mathrm{P}_{2} \mathrm{O}_{5} / \mathrm{ha}\right.$ ) of available phosphorus was observed in village Nemera Panchayat while the minimum value $(15 \mathrm{Kg}$ $\mathrm{P}_{2} \mathrm{O}_{5} /$ ha) was observed in Machwa Panchayat, respectively. Adopting the Muhr et al., (1965) classification, 25 samples (29.06\%) were found low ( $\left.<20 \mathrm{~kg} \mathrm{P}_{2} \mathrm{O}_{5} / \mathrm{ha}\right)$ and 61 samples $(70.93 \%)$ were found medium $(20-50 \mathrm{~kg}$ $\mathrm{P}_{2} \mathrm{O}_{5} / \mathrm{ha}$ ) in available phosphorus and none of the sample was found high $\left(>50 \mathrm{P}_{2} \mathrm{O}_{5} /\right.$ ha), i.e. soils were low to medium in available phosphorus. The farmers are using only diammonium phosphate (DAP) as a source of nutrients in adequate quantity. As a result, $\mathrm{P}$ is available in low to medium range. The soils are characterized by high fixation capacities resulting mainly on account of calcareous nature. Available $\mathrm{P}$ showed significant positive correlation with available $\mathrm{K}(\mathrm{r}=$ $\left.0.915^{* *}\right)$. The available potassium in these soils ranged between 205 and $360 \mathrm{~kg} \mathrm{~K} 2 \mathrm{O} / \mathrm{ha}$. 


\section{Int.J.Curr.Microbiol.App.Sci (2017) 6(3): 88-96}

Table.1 Fertility status of soils of Jhotwar Panchayat Samiti of Jaipur district

\begin{tabular}{|c|c|c|c|c|c|c|c|c|c|c|c|c|c|}
\hline $\begin{array}{l}\text { S. } \\
\text { No } \\
\end{array}$ & $\begin{array}{c}\text { Name of } \\
\text { panchayat }\end{array}$ & $\begin{array}{c}\text { No.of } \\
\text { sample }\end{array}$ & pH & $\begin{array}{c}\mathbf{E C} \\
\left(\mathbf{d S m}^{-1}\right)\end{array}$ & OC (percent) & $\begin{array}{c}\text { Av. N } \\
(\mathrm{kg} / \mathrm{ha})\end{array}$ & $\begin{array}{c}\text { Av. } \\
\text { P(kg/ha) }\end{array}$ & $\begin{array}{c}\text { Av. } \\
\text { K(kg/ha) }\end{array}$ & $\begin{array}{c}\text { Av. S } \\
\left(\mathrm{mg} \mathrm{kg}^{-1}\right.\end{array}$ & $\begin{array}{c}\text { Av. Zn } \\
\left(\mathrm{mg} \mathrm{kg}^{-1}\right)\end{array}$ & $\begin{array}{c}\text { Av. Fe } \\
\left(\mathrm{mg} \mathrm{kg}^{-1}\right)\end{array}$ & $\begin{array}{c}\mathbf{A v} \cdot \mathbf{C u} \\
\left(\mathrm{mg} \mathrm{kg}^{-1}\right)\end{array}$ & $\begin{array}{r}\text { Av. Cu } \\
\left(\mathrm{mg} \mathrm{kg}^{-1}\right)\end{array}$ \\
\hline 1 & Pachar & 3 & $\begin{array}{c}7.50-7.80 \\
(7.63)\end{array}$ & $\begin{array}{c}1.20-1.81 \\
(1.57)\end{array}$ & $\begin{array}{l}0.24-0.38 \\
(0.33)\end{array}$ & $\begin{array}{c}150.25-191.20 \\
(176.54)\end{array}$ & $\begin{array}{c}23-43 \\
(35.33)\end{array}$ & $\begin{array}{l}255-339 \\
(310.33)\end{array}$ & $\begin{array}{c}6.85-10.85 \\
(9.38)\end{array}$ & $\begin{array}{c}0.28-0.56 \\
(0.45)\end{array}$ & $\begin{array}{l}2.60-2.87 \\
(2.75)\end{array}$ & $\begin{array}{l}0.28-0.51 \\
\quad(0.42)\end{array}$ & $\begin{array}{l}3-4.42 \\
(3.92)\end{array}$ \\
\hline 2 & Kalwar & 4 & $\begin{array}{c}7.50-8.30 \\
(7.8)\end{array}$ & $\begin{array}{l}0.85-2.00 \\
(1.56)\end{array}$ & $\begin{array}{l}0.14-0.38 \\
(0.25)\end{array}$ & $\begin{array}{c}130.93-190.52 \\
(159.23)\end{array}$ & $\begin{array}{c}20-43 \\
(29.85)\end{array}$ & $\begin{array}{l}225-338 \\
(159.23)\end{array}$ & $\begin{array}{c}4.80-10.57 \\
(7.54)\end{array}$ & $\begin{array}{c}0.30-0.54 \\
(0.37)\end{array}$ & $\begin{array}{l}1.50-2.82 \\
(2.13)\end{array}$ & $\begin{array}{l}0.20-0.49 \\
(0.33)\end{array}$ & $\begin{array}{l}1.58-4.40 \\
(2.89)\end{array}$ \\
\hline 3 & Bhambhori & 3 & $\begin{array}{c}7.90-8.10 \\
(8)\end{array}$ & $\begin{array}{c}0.95-1.40 \\
(1.11)\end{array}$ & $\begin{array}{c}0.15-0.31 \\
(0.2)\end{array}$ & $\begin{array}{c}133.07-170.20 \\
(144.72)\end{array}$ & $\begin{array}{c}23-33 \\
(25)\end{array}$ & $\begin{array}{c}220-320 \\
(254)\end{array}$ & $\begin{array}{l}4.77-8.40 \\
(6.19)\end{array}$ & $\begin{array}{c}0.18-0.40 \\
(0.26)\end{array}$ & $\begin{array}{c}1.40-2.49 \\
(1.75)\end{array}$ & $\begin{array}{c}0.23-0.35 \\
(0.27)\end{array}$ & $\begin{array}{c}1.68-3.65 \\
(2.37)\end{array}$ \\
\hline 4 & Machwa & 5 & $\begin{array}{c}7.90-8.30 \\
(8.06)\end{array}$ & $\begin{array}{c}0.92-1.90 \\
(1.38)\end{array}$ & $\begin{array}{c}0.11-0.18 \\
(0.18)\end{array}$ & $\begin{array}{c}125.43-140.10 \\
(140.03)\end{array}$ & $\begin{array}{l}15-22 \\
(22.4)\end{array}$ & $\begin{array}{c}205-230 \\
(244.2)\end{array}$ & $\begin{array}{l}3.53-5.20 \\
(6.12)\end{array}$ & $\begin{array}{c}0.17-0.20 \\
(0.22)\end{array}$ & $\begin{array}{c}1.30-1.42 \\
(1.66)\end{array}$ & $\begin{array}{l}0.21-0.25 \\
\quad(0.27)\end{array}$ & $\begin{array}{l}1.67-1.71 \\
(2.28)\end{array}$ \\
\hline 5 & Hathoj & 3 & $\begin{array}{c}7.90-8.00 \\
(7.97)\end{array}$ & $\begin{array}{c}1.09-1.39 \\
(1.23)\end{array}$ & $\begin{array}{l}0.15-0.27 \\
(0.22)\end{array}$ & $\begin{array}{c}135.16-160.98 \\
(148.08)\end{array}$ & $\begin{array}{c}20-27 \\
(23.33)\end{array}$ & $\begin{array}{l}225-203 \\
(276.33)\end{array}$ & $\begin{array}{l}6.25-8.20 \\
\quad(7.3)\end{array}$ & $\begin{array}{l}0.19-0.34 \\
(0.28)\end{array}$ & $\begin{array}{l}1.75-2.40 \\
(1.99)\end{array}$ & $\begin{array}{l}0.24-0.35 \\
(0.30)\end{array}$ & $\begin{array}{c}1.87-2.95 \\
(2.59)\end{array}$ \\
\hline 6 & Dhankiya & 10 & $\begin{array}{c}7.20-8.50 \\
(7.89)\end{array}$ & $\begin{array}{c}0.67-2.20 \\
(1.45)\end{array}$ & $\begin{array}{l}0.14-0.39 \\
(0.27)\end{array}$ & $\begin{array}{c}137.52-194.42 \\
(166.19)\end{array}$ & $\begin{array}{l}16-44 \\
(29.8)\end{array}$ & $\begin{array}{c}230-352 \\
(293.9)\end{array}$ & $\begin{array}{c}4.91-11.10 \\
(8.22)\end{array}$ & $\begin{array}{l}0.17-0.58 \\
(0.37)\end{array}$ & $\begin{array}{l}1.28-3.87 \\
(2.27)\end{array}$ & $\begin{array}{l}0.21-0.51 \\
(0.36)\end{array}$ & $\begin{array}{c}1.89-4.45 \\
(3.30)\end{array}$ \\
\hline 7 & Chakbasdi & 7 & $\begin{array}{c}7.60-8.50 \\
(8.21)\end{array}$ & $\begin{array}{c}0.53-1.30 \\
(0.90)\end{array}$ & $\begin{array}{c}0.17-0.32 \\
(0.27)\end{array}$ & $\begin{array}{c}138.84-175.48 \\
(162.08)\end{array}$ & $\begin{array}{l}21-33 \\
(29)\end{array}$ & $\begin{array}{l}260-344 \\
(305.57)\end{array}$ & $\begin{array}{l}5.20-10.30 \\
(7.78)\end{array}$ & $\begin{array}{l}0.24-0.42 \\
(0.35)\end{array}$ & $\begin{array}{l}1.99-2.42 \\
(2.27)\end{array}$ & $\begin{array}{l}0.23-0.37 \\
(0.31)\end{array}$ & $\begin{array}{l}2.20-3.70 \\
(3.09)\end{array}$ \\
\hline 8 & Niwarro & 5 & $\begin{array}{c}7.70-8.00 \\
(7.86)\end{array}$ & $\begin{array}{c}1.85-1.35 \\
(1.61)\end{array}$ & $\begin{array}{l}0.12-0.34 \\
\quad(0.27)\end{array}$ & $\begin{array}{c}128.57-182.48 \\
(165.07)\end{array}$ & $\begin{array}{l}18-36 \\
(29.6)\end{array}$ & $\begin{array}{c}225-345 \\
(303.6)\end{array}$ & $\begin{array}{l}4.88-10.11 \\
\quad(8.17)\end{array}$ & $\begin{array}{l}0.18-0.46 \\
(0.36)\end{array}$ & $\begin{array}{l}1.19-2.50 \\
(2.07)\end{array}$ & $\begin{array}{l}0.27-0.41 \\
(0.33)\end{array}$ & $\begin{array}{l}2.88-3.80 \\
(3.24)\end{array}$ \\
\hline 9 & Maheshwas & 4 & $\begin{array}{c}7.80-8.20 \\
(8.03)\end{array}$ & $\begin{array}{c}0.78-1.08 \\
(1.12)\end{array}$ & $\begin{array}{l}0.15-0.20 \\
(0.17)\end{array}$ & $\begin{array}{c}134.52-147.52 \\
(139.84)\end{array}$ & $\begin{array}{l}18-23 \\
(20.75)\end{array}$ & $\begin{array}{l}225-268 \\
(243.75)\end{array}$ & $\begin{array}{l}4.80-6.30 \\
(5.31)\end{array}$ & $\begin{array}{c}0.18-0.22 \\
(0.2)\end{array}$ & $\begin{array}{c}1.75-1.87 \\
(1.81)\end{array}$ & $\begin{array}{l}0.22-0.27 \\
(0.25)\end{array}$ & $\begin{array}{l}2.10-2.25 \\
(2.19)\end{array}$ \\
\hline 10 & Begus & 8 & $\begin{array}{c}8.10-8.50 \\
(8.35)\end{array}$ & $\begin{array}{c}0.55-1.05 \\
(0.80)\end{array}$ & $\begin{array}{c}0.10-0.24 \\
(0.14)\end{array}$ & $\begin{array}{c}122.07-150.50 \\
(133.03)\end{array}$ & $\begin{array}{l}16-23 \\
(18.5)\end{array}$ & $\begin{array}{l}212-255 \\
(238.75)\end{array}$ & $\begin{array}{l}3.53-7.10 \\
(4.53)\end{array}$ & $\begin{array}{c}0.17-0.30 \\
(0.21)\end{array}$ & $\begin{array}{l}1.19-2.47 \\
(1.54)\end{array}$ & $\begin{array}{l}0.20-0.31 \\
(0.23)\end{array}$ & $\begin{array}{c}1.10-2.75 \\
(1.72)\end{array}$ \\
\hline 11 & Nemera & 6 & $\begin{array}{c}7.40-8.30 \\
(7.88)\end{array}$ & $\begin{array}{l}0.83-2.20 \\
(1.4)\end{array}$ & $\begin{array}{l}0.16-0.40 \\
(0.27)\end{array}$ & $\begin{array}{c}138.12-198.71 \\
(161.74)\end{array}$ & $\begin{array}{l}20-47 \\
(30.5)\end{array}$ & $\begin{array}{l}254-360 \\
(305.83)\end{array}$ & $\begin{array}{c}4.90-11.45 \\
(7.9)\end{array}$ & $\begin{array}{l}0.21-0.56 \\
(0.36)\end{array}$ & $\begin{array}{l}1.85-4.10 \\
(2.59)\end{array}$ & $\begin{array}{l}0.22-0.51 \\
(0.34)\end{array}$ & $\begin{array}{c}2.15-4.65 \\
(3.26)\end{array}$ \\
\hline 12 & Mundiaramsar & 11 & $\begin{array}{c}7.50-8.30 \\
(7.96)\end{array}$ & $\begin{array}{c}0.85-1.88 \\
(1.25)\end{array}$ & $\begin{array}{l}0.14-0.35 \\
(0.25)\end{array}$ & $\begin{array}{c}137.98-185.34 \\
(159.70)\end{array}$ & $\begin{array}{c}16-40 \\
(27.27)\end{array}$ & $\begin{array}{l}225-346 \\
(281.18)\end{array}$ & $\begin{array}{l}4.95-10.45 \\
\quad(7.52)\end{array}$ & $\begin{array}{c}0.17-0.52 \\
(0.32)\end{array}$ & $\begin{array}{c}1.29-3.61 \\
(2.30)\end{array}$ & $\begin{array}{c}0.20-0.45 \\
(0.32)\end{array}$ & $\begin{array}{c}2.10-4.39 \\
(3.02)\end{array}$ \\
\hline 13 & Durjiniawas & 8 & $\begin{array}{c}7.50-8.50 \\
(8.11)\end{array}$ & $\begin{array}{c}0.55-2.10 \\
(1.16)\end{array}$ & $\begin{array}{c}0.14-0.37 \\
(0.26)\end{array}$ & $\begin{array}{c}135.02-190.20 \\
(162.39)\end{array}$ & $\begin{array}{l}20-43 \\
(29.5)\end{array}$ & $\begin{array}{l}225-349 \\
(295.88)\end{array}$ & $\begin{array}{l}4.60-10.85 \\
(7.66)\end{array}$ & $\begin{array}{c}0.18-0.56 \\
(0.36)\end{array}$ & $\begin{array}{c}1.50-2.90 \\
(2.23)\end{array}$ & $\begin{array}{c}0.23-0.51 \\
(0.35)\end{array}$ & $\begin{array}{c}2.05-4.75 \\
(3.34)\end{array}$ \\
\hline 14 & Sumel & 9 & $\begin{array}{c}7.30-8.40 \\
(7.94)\end{array}$ & $\begin{array}{c}0.77-2.51 \\
(1.41)\end{array}$ & $\begin{array}{c}0.14-0.39 \\
(0.28)\end{array}$ & $\begin{array}{c}132.52-190.16 \\
(162.79)\end{array}$ & $\begin{array}{l}18-43 \\
(30.56)\end{array}$ & $\begin{array}{l}245-357 \\
(295.78)\end{array}$ & $\begin{array}{c}4.77-11.50 \\
(7.93)\end{array}$ & $\begin{array}{c}0.18-0.58 \\
(0.37)\end{array}$ & $\begin{array}{c}1.59-4.10 \\
(2.61)\end{array}$ & $\begin{array}{c}0.23-0.55 \\
(0.35)\end{array}$ & $\begin{array}{c}2.30-4.67 \\
(3.57)\end{array}$ \\
\hline & $\begin{array}{l}\text { Range Overall } \\
\text { mean }\end{array}$ & - & 8 & 1.27 & 0.24 & 156.76 & 27.40 & 282.63 & 7.39 & 0.32 & 2.17 & 0.32 & 2.96 \\
\hline
\end{tabular}

Value in Parenthesis shows mean 
Table.2 Correlation matrix for various soil properties

\begin{tabular}{|c|c|c|c|c|c|c|c|c|c|c|c|}
\hline & pH & EC & $\begin{array}{c}\text { Organic } \\
\text { carbon } \\
\text { (percent) }\end{array}$ & $\begin{array}{c}\text { Available } \\
\text { N } \\
\text { (kg/ha) }\end{array}$ & $\begin{array}{c}\text { Available } \\
\left(\mathrm{P}_{2} \mathrm{O}_{5}\right) \\
\text { kg/ha }\end{array}$ & $\begin{array}{c}\text { Available } \\
\left(\mathrm{K}_{2} \mathrm{O}\right) \\
\text { kg/ha }\end{array}$ & $\begin{array}{c}\text { Available } \\
\text { S } \\
\left(\mathrm{mg} \mathrm{kg}^{-1}\right)\end{array}$ & $\begin{array}{c}\text { Available } \\
\mathrm{Zn} \\
\left(\mathrm{mg} \mathrm{kg}^{-1}\right)\end{array}$ & $\begin{array}{c}\text { Available } \\
\text { Fe } \\
\left(\mathrm{mg} \mathrm{kg}^{-1}\right)\end{array}$ & $\begin{array}{c}\text { Available } \\
\qquad \mathrm{Cu} \\
\left(\mathrm{mg} \mathrm{kg}^{-1}\right)\end{array}$ & $\begin{array}{c}\text { Available } \\
\text { Mn } \\
\left(\mathrm{mg} \mathrm{kg}^{-1}\right)\end{array}$ \\
\hline $\mathrm{pH}$ & 1.000 & $\begin{array}{c}- \\
0.929 * *\end{array}$ & $-0.435 * *$ & $-0.430 * *$ & $-0.449 * *$ & $-0.335 * *$ & $-0.434 * *$ & $-0.448 * *$ & $-0.423 * *$ & $-0.461 * *$ & $-0.477 * *$ \\
\hline $\mathrm{EC}$ & & 1.000 & $0.437 * *$ & $0.430 * *$ & $0.467 * *$ & $0.356 * *$ & $0.451 * *$ & $0.447 * *$ & $0.395 * *$ & $0.479 * *$ & $0.492 * *$ \\
\hline $\mathrm{OC}$ & & & $1.000 * *$ & $0.989 * *$ & $0.970 * *$ & $0.920 * *$ & $0.968 * *$ & $0.965 * *$ & $0.864 * *$ & $0.924 * *$ & $0.950 * *$ \\
\hline $\mathrm{N}$ & & & & 1.000 & $0.974 * *$ & $0.924 * *$ & $0.971 * *$ & $0.971 * *$ & $0.845^{* *}$ & $0.931 * *$ & $0.940 * *$ \\
\hline $\mathrm{P}_{2} \mathrm{O}_{5}$ & & & & & 1.000 & $0.915^{* *}$ & $0.960 * *$ & $0.973^{* *}$ & $0.849 * *$ & $0.943^{* *}$ & $0.938^{* *}$ \\
\hline $\mathrm{K}_{2} \mathrm{O}$ & & & & & & 1.000 & $0.922 * *$ & $0.929 * *$ & $0.810 * *$ & $0.853 * *$ & $0.884 * *$ \\
\hline $\mathrm{S}$ & & & & & & & 1.000 & $0.951 * *$ & $0.830 * *$ & $0.943^{* *}$ & $0.932 * *$ \\
\hline $\mathrm{Zn}$ & & & & & & & & 1.000 & $0.848 * *$ & $0.934 * *$ & $0.927 * *$ \\
\hline $\mathrm{Fe}$ & & & & & & & & & 1.000 & $0.825^{* *}$ & $0.828 * *$ \\
\hline $\mathrm{Cu}$ & & & & & & & & & & 1.000 & $0.920 * *$ \\
\hline $\mathrm{Mn}$ & & & & & & & & & & & 1.000 \\
\hline
\end{tabular}

** significant at 0.01 per cent level of significance 
The lowest value of available potassium was observed in the soils samples collected from Machwa Panchayat, whereas the highest value was found in the sample of Nemera Panchayat. As per criterion laid down by Muhr et al., (1965), 40 soils are under medium category (125 to $300 \mathrm{~kg} \mathrm{~K} 2 \mathrm{O} / \mathrm{ha}$ ) and 46 soils samples fall under high category (above $300 \mathrm{~kg} \mathrm{~K}_{2} \mathrm{O} / \mathrm{ha}$ ) available potassium. The average available $\mathrm{S}$ content in the surface soil samples varied from 3.53 to $11.50 \mathrm{mg} \mathrm{kg}$ ${ }^{1}$ with a mean value of $7.39 \mathrm{mgkg}^{-}$ ${ }^{1}$.Considering $10 \mathrm{mg} \mathrm{kg}^{-1}$ as critical limits for available $\mathrm{S}$ have been given by (Tandon, 1992). Among the 86 surface soil sample analyzed, the lowest value was observed in Begus Panchayat and the highest value was found in Sumel Panchayat. Such results were also reported by Jat and Yadav (2006). The availability of phosphorus also increased with increase in organic carbon which might be due to, (i) formation of phosphorus humic complexes which are easily assimilated by plants, (ii) anions replacement of phosphate by humation and (iii) the coating of sesquioxide by particles of humus to form a protective cover and thus reduce the phosphorus fixing capacity of the soils (Gharu and Tarafdar, 2004).

\section{Available micronutrients}

The data presented in the table 1. The available copper content of surface soils was ranged between 1.10 to $4.75 \mathrm{mg} \mathrm{kg}^{-1}$ with mean value of $2.96 \mathrm{mg} \mathrm{kg}^{-1}$. The minimum (1.10 $\mathrm{mg} \mathrm{kg}^{-1}$ ) in Begus Panchayat and the maximum $\left(4.75 \mathrm{mg} \mathrm{kg}^{-1}\right.$ ) contents of available copper were recorded in Durjiniwas Panchayat, Kalwar, Chakmogia and Malpurachor, respectively. The soils of Jhotwara panchyat samiti were found 93.02 per cent soils samples sufficient in available copper content as per critical limit suggested by Lindsay and Norvell (1978). Such findings for available copper of soils of Nagaur and Jodhpur were also suggested by Joshi and
Dhir (1983). The availability and supply of manganese to soil plant system is governed by oxidation and reduction processes that are influenced by number of factors. A close study of data pertaining to available manganese (Table 1) reveals that the available manganese content in soils ranged from 0.20 to $0.55 \mathrm{mg} \mathrm{kg}^{-1}$ with mean value of $0.32 \mathrm{mg}$ $\mathrm{kg}^{-1}$. The minimum available manganese $\left(0.20 \mathrm{mg} \mathrm{kg}^{-1}\right)$ was recorded in soil sample of Mundiaramsar and Kalwar Panchayat while the maximum $\left(4.75 \mathrm{mg} \mathrm{kg}^{-1}\right)$ was found in the soil sample of Sumle. In general, soils of Jhotwara Panchyat samiti, 84.88 percent soils samples are sufficient in available manganese (as per critical limit suggested by Lindsay and Norvell, 1978). Such results were also reported by Sharma et al., (2003).The available Fe content of surface soil samples varied from 1.19 to $2.87 \mathrm{mg} \mathrm{kg}^{-1}$ with a mean value of $2.17 \mathrm{mg} \mathrm{kg}^{-1}$. The minimum available iron (1.19 mg kg $\mathrm{mg}^{-1}$ was recorded in soil sample of Begus and Niwaroo Panchayat while the maximum iron content $\left(4.10 \mathrm{mg} \mathrm{kg}^{-}\right.$ $\left.{ }^{1}\right)$ was recorded in soil sample of Pachar Panchayat. On the basis of critical limit of available iron, suggested by Lindsay and Norvell (1978). Such results were also reported by Singh et al., (2008). The 97.67 per cent soils samples of Jhotwara Panchayat Samiti were found deficient in available iron content. The average $\mathrm{Zn}$ content of surface sample ranges from 0.17 to $0.58 \mathrm{mg} \mathrm{kg}^{-1}$ with a mean value of $0.32 \mathrm{mg} \mathrm{kg}^{-1}$. As a whole, the lowest value was observed in Panchayat of Begus, Dhankiya and Mundiaramsar whereas, the highest value was found in Sumel Panchayat. Zinc was found to be deficient in the entire study area). Such results were also reported by Jethra et al., (1993). The study areas soils are general low in available $\mathrm{N}$, low to medium in available $\mathrm{P}$, high in available $\mathrm{K}$ and low in available $S$. The soils of available micronutrient were found to be well supplied with $\mathrm{Mn}$ and $\mathrm{Cu}$ and deficient in $\mathrm{Fe}$ and $\mathrm{Zn}$ supply. 
In conclusion, the result of fertility status of irrigated soils of Jhotwara-panchayat samiti Jaipur district (Rajasthan) indicated that among the 86 samples analyzed, soils were low in organic carbon ( 0.10 to 0.40 percent) and available nitrogen (122.07 to $198.71 \mathrm{~kg}$ $\mathrm{ha}^{-1}$ ), whereas low to medium in available phosphorus (15 to $47 \mathrm{~kg} \mathrm{P}_{2} \mathrm{O}_{5} \mathrm{ha}^{-1}$ ) and available sulphur (3.53 to $11.50 \mathrm{mg} \mathrm{kg}^{-1}$ ) and medium to high in available potassium (205 to $360 \mathrm{~kg} \mathrm{~K}_{2} \mathrm{O} \mathrm{ha}{ }^{-1}$ ). The micronutrients are mostelyzn and Fe deficient.

\section{References}

Gharu, A. and Tarafdar, J.C. 2004. Influence of organic acids on mobilization of inorganic and organic phosphorus in soil. J. Indian Society of Soil Sci., 24: 248-253.

Gupta, K. 2003. Available micronutrient status and their effect on soil properties of Nagaur (Rajasthan. M.Sc. Ag.) Thesis, Rajasthan Agricultural University, Bikaner.

Jat, J.R. and Yadav, B.L. 2006. Different forms of $S$ and their relationship with properties of Entisols of Jaipur district (Rajasthan) under mustard cultivation. J. Indian Society of Soil Sci., 54: 208212.

Jethra, J.K., Khandelwal, R.B. and Musin, M.R. 1993. Available micronutrient status in semi-arid eastern plain zone of Rajasthan. $58^{\text {th }}$ Annual convention of ISSS held from October 8-12, 1993. $J$. Indian Society of Soil Sci., 41: 156-158.

Jackson, M.L. 1973. Soil Chemical Analysis. Prentice Hall of India Pvt. Ltd, New Delhi.

Jolivet, C., Arrouays, D. Andreux, F. and Leveque, J. 1997. Soil organic carbon dynamic in cleared temperate forest spodosols converted to maize cropping. Plant and Soil, 191: 225-231.

Joshi, D.C. and Dhir, R.P. 1983. Available forms of $\mathrm{Mn}$ and $\mathrm{Fe}$ in some arid soils and their relation with soils properties. Annals of Arid Zone, 22: 7-14.

Lindsay, W.L. and Norvell, W.A. 1978. Development of DTPA soil test for zinc, iron, manganese and copper. Soil Sci. Society America Proceeding, 42: 421- 428.

Meena, H.B., Sharma, P.R. and Rawat, U.S. 2006. Status of macromicronutrients in some soils of Tonk district of Rajasthan. J. Indian Society of Soil Sci., 54: 508 512.

Mathur, G.M., Ramdev and Yadav, B.S. 2006. Status of zinc in irrigated NorthWest plain soils of Rajasthan. J. Indian Society of Soil Sci., 54: 359-361

Muhr, G.R., Datta, N.P., Shankar Subramany, N., Dever, F., Lecy, V.K. and Donahue, R.R. 1965. Soil testing in India, USDA Publication., pp. 120.

Olsen, S.R., Cole, R.V., Watanabe, F.S. and Dean, L.A. 1954. Estimation of available phosphorus in soil by extraction with sodium bicarbonate. Circular USDA, No. 939.

Sharma, R.P., Singh, M. and Sharma, J.P. 2003. Correlation studies on micronutrients vis-à-vis soil properties in some soils of Nagaur district in semi arid region of Rajasthan. J. Indian Society of Soil Sci., 51(4): 522-527.

Singh, M. and Singh, K.S. 1981. Zinc and copper status of soils of Rajasthan. Annals of Arid Zone, 20: 77-85.

Subbiah, B.V. and Asija, G.L. 1956. A rapid procedure for the estimation of available nitrogen in Soil, Curr. Sci., 25: 259-260.

Summer, M.E. 1995. Sodic soils New perspective. In Australian sodic soils distribution, properties and management (R. Naidu. M.E. Summer and P. Rengasamy Eds.) pp.1-34. CSIRO, Australian.

Sharma, S.S., Totawat, K.L. and Shyampura, 
R.L. 2004. Characterization and classification of salt affected soils of Southern Rajasthan. J. Indian Society of Soil Sci., 52: 209-14.

Singh, R.S., Dubey, P.N., Singh, S.K. and Shyampura, R.L. 2008. Distribution of chemical fractions of micronutrient cations in some vertisols under the Agro-Eco-Sub Region 4.2 of Eastern Rajastan. J. Indian Society of Soil Sci., 56: 192-197.

Sharma, P.K., Anil Sood, Setia, R.K., Tur, N.S., Deepak Mehra, and Singh, H. 2008. mapping of macronutrients in soils of Amritsar district (punjab)- a GIS approach. J. Indian Society of Soil Sci., 56: 34-41.

Tandon, H.L.S. 1992. Fertilizer guide. Fertilizers Development and Consultation Organization, New Delhi. Verma, L.P., Tripathi, B.R. and Sharma, D.P.
1980. Organic carbon as a inden to assess the nitrogen status of the soils. $J$. Indian Society of Soil Sci., 28: 138-140.

Walkley, A. and Black, I.A. 1934. Rapid titration method of organic carbon of soils. Soil Sci., 37: 29-33.

Williams, C.H. and Steinbergs, A. 1959. Soil sulphur interactions as chemical indices of available sulphur in some Australian soils. Australian J. Agri. Res., 10: 340352.

Wu, R. and Tiessen, H. 2002. Effact of land use on soil degradation in alpine grassland soil China, Soil Sci. Society of the America J., 66: 1648-1656.

Yadav, R.L. and Meena, M.C. 2009. Available micronutrient status and their relationship with soil properties of Degana soil series of Rajasthan. $J$. Indian Society of Soil Sci., 57: 90-92.

\section{How to cite this article:}

Kamla Kumari Choudhary, B.L. Yadav, K.K. Sharma, R.D. Jat and Kakraliya, S.K. 2017. Fertility Status of Irrigated Soils of Jhotwara Panchayat Samiti of Jaipur District. Int.J.Curr.Microbiol.App.Sci. 6(3): 88-96. doi: https://doi.org/10.20546/ijcmas.2017.603.009 